\title{
APLICAÇÃO DE TESTE DE BIODEGRABILIDADE PARA SELEÇÃO DE CONSÓRCIO FÚNGICO PROMISSOR NA DEGRADAÇÃO DE HIDROCARBONETOS DE PETRÓLEO
}

\author{
S. D. da ROCHA ${ }^{1}$, L. M. de CASTRO ${ }^{1}$, J. R. S. de SOUZA ${ }^{2}$, T. C. S. de OLIVEIRA ${ }^{1}$ \\ ${ }^{1}$ Universidade Federal do Amazonas, Departamento de Química \\ ${ }^{2}$ Universidade Federal do Amazonas, Departamento de Biologia \\ E-mail para contato: sissarocha26@gmail.com
}

\begin{abstract}
RESUMO - Pesquisas têm demonstrado que a ampla gama de comunidades microbianas nativas da região Amazônica apresentam grupos promissores com grande interesse biotecnológico. Este trabalho teve como finalidade selecionar um consórcio microbiano composto por fungos isolados a partir da macrófita Eichhornia crassipes Mart. Solms. Para tanto, foi empregado um teste de biodegradabilidade utilizando-se o indicador redox 2,6-diclorofenol indofenol (DCPIP), que indica a ocorrência de oxidação biológica através da mudança da coloração do meio de cultivo de azulado para transparente. A escolha do consórcio baseou-se na velocidade de degradação dos contaminantes em até 76 horas de experimento. De acordo com os ensaios, os fungos codificados: F01, F06, F13, F14, F19, F23 e F27 apresentaram tempo de degradação do petróleo bruto em até 48 horas, o que indica que os mesmos podem constituir consórcios fúngicos promissores para tratamento de água por biorremediação.
\end{abstract}

\section{INTRODUÇÃO}

A poluição ambiental causada por hidrocarbonetos de petróleo e seus derivados tem recebido destaque nas últimas décadas e causado uma intensa mobilização de empresas privadas e do poder público em todo o mundo (Tonini et al., 2010). Nesse contexto tem aumentado a demanda de estudo de caracterização de microrganismos para sua aplicação na biorremediação de áreas degradadas.

O petróleo é constituído por diferentes classes de hidrocarbonetos. Por esse fato, a degradação de seus compostos não pode ser realizada por uma única espécie microbiana, sendo necessária a formação de consórcios por espécies distintas. Por complementaridade metabólica, os consórcios degradam os compostos derivados do petróleo, podendo até mesmo chegar à sua completa mineralização. Vale destacar que a utilização de vários microrganismos melhoram a eficiência da biorremediação (Leahy e Colwell, 1990; Van Hamme et al., 2000; Crapez et al., 2002; Ghazali et al., 2004; Jacques et al., 2007).

A constante presença do petróleo e seus derivados em uma mesma localidade aumenta a variedade dos microrganismos que podem servir como indicadores biológicos de ambientes impactados, conforme Peixoto et al. (2008). Os fungos filamentosos em geral, são considerados bons degradadores, pois devido ao seu crescimento micelial, eles ramificam-se 
rapidamente no substrato, digerindo-o por meio da secreção de enzimas extracelulares, disponibilizando, desta forma, o acesso para o ataque de bactérias.

O teste de biodegrabilidade pelo uso do indicador redox 2,6-diclorofenol indofenol (DCPIP) foi desenvolvido por Hanson et al. (1993). O princípio desta técnica é que durante a oxidação microbiana dos hidrocarbonetos, elétrons são transferidos até aceptores como oxigênio, nitrato e sulfato. Ao incorporar um aceptor de elétron como o DCPIP ao meio de cultura, é possível averiguar a capacidade dos microrganismos em utilizar hidrocarbonetos como substrato pela observação da mudança de cor do DCPIP de azul (oxidado) para incolor (reduzido). O emprego de fungos filamentosos neste trabalho deve-se a um aspecto de especial interesse que é a tolerância dos mesmos em ambientes contaminados e tóxicos. Acredita-se que através da compreensão do poder de ação de microrganismos degradadores dos grupos de hidrocarbonetos, seja possível ampliar este potencial em escala industrial de modo a encontrar alternativas viáveis para a descontaminação, em especial de ambientes aquáticos.

\section{METODOLOGIA}

\subsection{Amostragem}

A seleção do consórcio fúngico envolveu 10 linhagens de fungos filamentosos isolados a partir da macrófita Eichhornia crassipes Mart. Solms que foi coletada no igarapé da Guarita (S0308'46.3” e O5957'24,4”), área que recebe a saída de efluentes da REMAN - Refinaria Isaac Sabbá da Petrobrás/Manaus-AM, conforme mostrado na Figura 1 (plotada com o auxílio do programa $R$ 3.1.2). Os fungos utilizados no experimento fazem parte da coleção de fungos filamentosos do Projeto Rede CTPetro Amazônia do Instituto de Pesquisas da Amazônia (INPA) em parceria com a Universidade Federal do Amazonas (UFAM).

Figura 1 - Mapa de localização do ponto de coleta da macrófita.

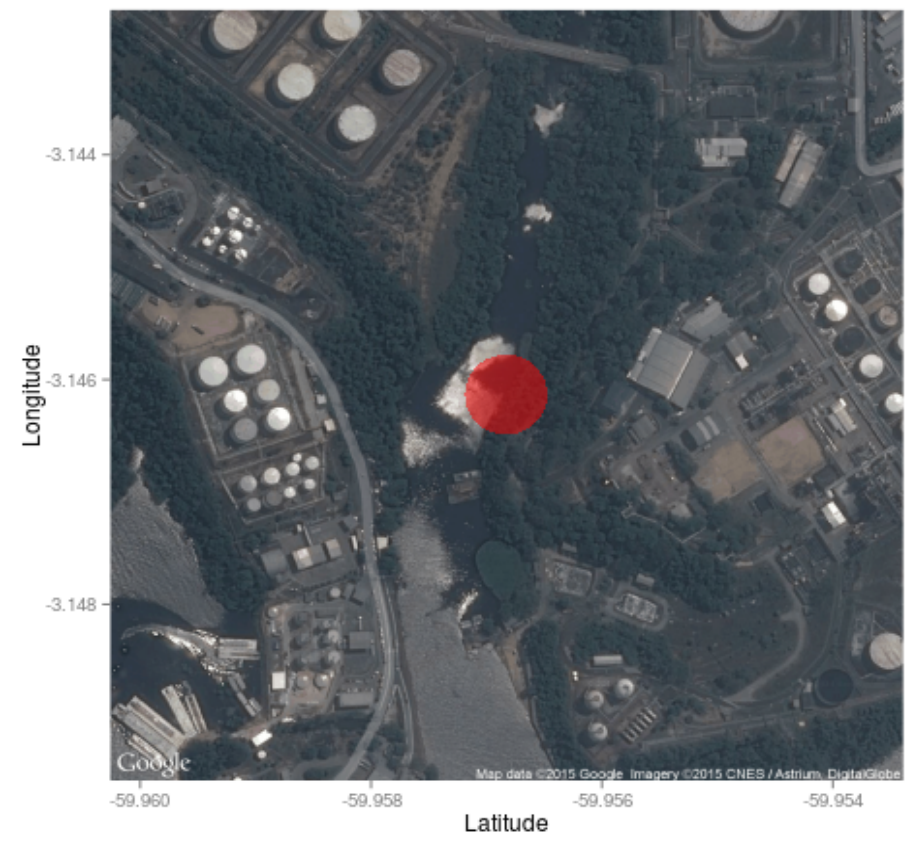




\subsection{Isolamento dos Fungos}

$\mathrm{O}$ isolamento de fungos foi realizado conforme a metodologia descrita por Pereira (1993). Após o crescimento dos fungos isolados, foram geradas culturas monospóricas a fim de se obter uma nova colônia de acordo com o protocolo desenvolvido por Azevedo e Costa (1973).

\subsection{Reativação dos Fungos}

Foram utilizadas 10 linhagens dos seguintes fungos: Fusarium, Penicillium, Bionectria e Phoma. Os fungos foram inoculados em meio de cultura BDA (Batata, Dextrose e Agar) acrescido com $30 \mu \mathrm{L}$ de petróleo bruto (proveniente da Base Petrolífera de Urucu - AM) e incubados em estufa D.B.O (demanda bioquímica de oxigênio) por um período de 7 dias a $28^{\circ} \mathrm{C}$.

\subsection{Teste de Biodegrabilidade}

Foi utilizada a técnica do indicador redox, 2,6-diclorofenol indofenol, por meio da ocorrência de oxidação biológica indicada pela mudança na coloração do meio de cultivo. Primeiramente, foi produzido um inóculo de cada fungo: uma alçada por $\mathrm{mL}$ de água esterilizada em um tubo de ensaio. Os tubos foram vedados com papel alumínio autoclavado e colocados para homogeneizar em um vórtex. Em seguida, foi adicionado $1 \mathrm{~mL}$ do inóculo de cada fungo em $20 \mathrm{~mL}$ de caldo Bushnell Haas acrescido de $100 \mu \mathrm{L}$ de petróleo bruto, $200 \mu \mathrm{L}$ de ampicilina $25 \mathrm{mg} \cdot \mathrm{mL}^{-1}$ e $0,5 \mathrm{~mL}$ do indicador redox DCPIP nos frascos. O teste foi desenvolvido em triplicata, sendo utilizados como controle os frascos contendo o meio com o petróleo, sem presença de fungos e outros contendo o meio com o fungo, porém sem o petróleo. Os frascos foram incubados em shaker com temperatura média a $30^{\circ} \mathrm{C}$ a $120 \mathrm{rpm}$ para analisar o crescimento dos fungos durante um período de 76 horas, ao final do qual foi realizado um comparativo entre a descoloração do indicador DCPIP obtida nos frascos inoculados com os controles. A Tabela 1 apresenta a identificação das espécies de fungos utilizadas no experimento.

Tabela 1 - Identificação dos fungos selecionados para testes de biodegrabilidade

\begin{tabular}{cc}
\hline Fungos & Espécies \\
\hline F01 & Penicillium citrinum \\
F06 & Phoma herbarum \\
F13 & Fusarium moniliforme \\
F14 & Phoma herbarum \\
F19 & Fusarium oxysporum \\
F23 & Phoma herbarum \\
F24 & Fusarium moniliforme \\
F27 & Penicillium sp. \\
F45 & Phoma herbarum \\
F46 & Bionectria ochroleuca \\
\hline
\end{tabular}


A análise qualitativa do teste do indicador redox DCPIP foi monitorada por meio da alteração de coloração dos meios de azul para incolor nos diferentes ensaios. Esta avaliação foi realizada por meio do acompanhamento visual a cada 8 horas com padrão de iluminação e registro fotográfico, conforme Figura 2.

Figura 2 - Amostras de fungos após a oxidação biológica, sinalizada pela mudança de cor do indicador DCPIP.

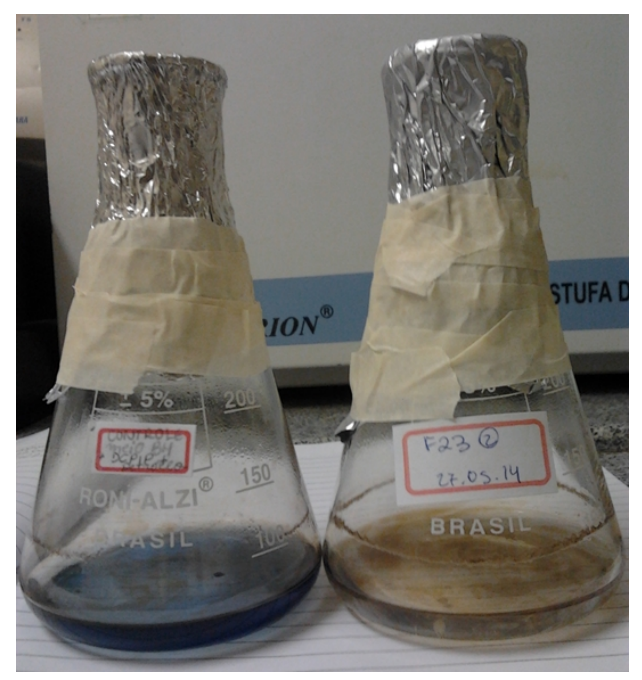

\section{RESULTADOS E DISCUSSÃO}

Os ensaios de biodegrabilidade demonstraram que os 10 fungos analisados possuem habilidade para degradar hidrocarbonetos de petróleo. Verificou-se a ocorrência de oxidação biológica em todas as amostras testadas com variação entre 24 a 76 horas de incubação no meio de cultivo a $30 \pm 1{ }^{\circ} \mathrm{C}$, sendo que a cinética da degradação do petróleo bruto ocorreu antes do tempo previsto que fora estimado em 7 dias.

O teste do DCPIP realizado por Bisognin (2012) demonstrou que 8 colônias de fungos isolados de uma biopilha apresentaram capacidade de biodegradar o contaminante sem a dependência de outras espécies. Esta avaliação permitiu constatar maior eficiência por determinadas colônias pertencentes aos gêneros Aspergillus, Trichoderma, Penicillium, Cladosporium e Verticillium. Também foi constatado no mesmo trabalho que em $80 \%$ dos arranjos de microrganismos avaliados, os consórcios microbianos apresentaram resultados satisfatórios mais rapidamente que as culturas puras na biodegradação do óleo diesel.

Araújo e Lemos (2002) identificaram 60 fungos filamentosos com capacidade em degradar hidrocarbonetos, os quais foram agrupados em quatro gêneros: Fusarium, Penicillium, Paecilomyces e Aspergillus. Os dois primeiros gêneros citados por esses autores também foram verificados neste trabalho e ambos obtiveram destaque no teste de biodegrabilidade. As bactérias também têm potencial para degradar hidrocarbonetos. Mariano (2006) utilizou o teste do indicador redox DCPIP para estudar a degradação de óleo diesel por consórcios das bactérias Staphylococcus hominis, Kocuria palustres e Pseudomonas aeruginosa, obtendo resultados positivos. 
O Gráfico 1, a seguir, foi criado com o auxílio do software Action 2.4, extensão do Programa Excel, e relaciona o tempo de degradação com os fungos selecionados, mostrando o potencial de degradação de petróleo para cada fungo.

Gráfico 1 - Relação do tempo de degradação versus fungos selecionados para verificação da ocorrência de oxidação biológica.

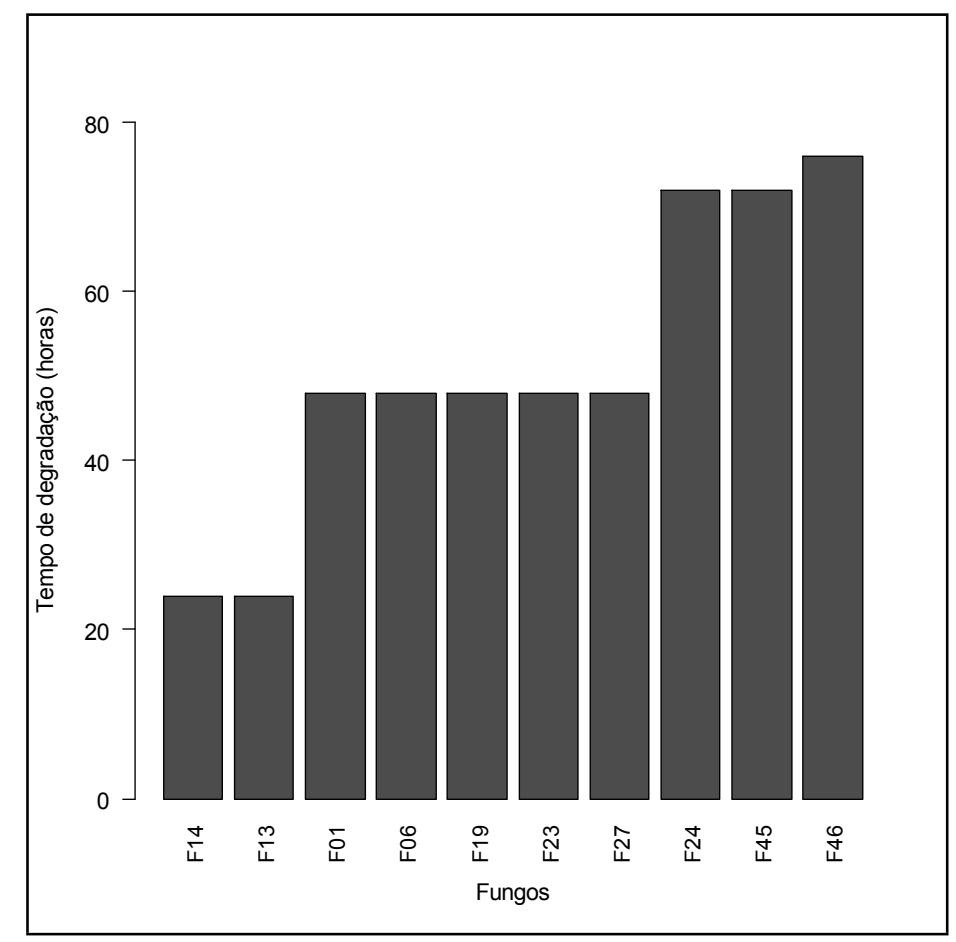

Por meio do gráfico observa-se que os fungos codificados: F13 e F14 foram os quais apresentaram maior eficiência de degradação biológica quanto à cinética da reação observando-se que o tempo de degradação foi de 24 horas. Observa-se que os fungos dos gêneros Penicillium (F01 e F27), Phoma (F06, F14, F23 e F45) e Fusarium (F13, F24 e F19) apresentaram maior eficiência na degradação biológica quanto à cinética da reação se comparados com o Bionectria (F46), o qual apresentou o maior tempo para degradação do petróleo, de $76 \mathrm{~h}$.

Considera-se que os fungos codificados F13, F14, F01, F06, F19, F23 e F27 apresentaram bons resultados como agentes degradadores de hidrocarbonetos de petróleo em até 48 horas de experimento. Tais espécies podem agir por complementaridade e liberar enzimas diferentes que ajudam no processo de conversão do contaminante em nutrientes, $\mathrm{CO}_{2}$ e $\mathrm{H}_{2} \mathrm{O}$. Portanto, a somatória do potencial destes fungos na produção do consórcio microbiano torna essas linhagens promissoras para possíveis tratamentos por biorremediação.

\section{CONSIDERAÇÕES FINAIS}

As linhagens de fungos utilizadas demonstraram potencial para a biodegradação de hidrocarbonetos de petróleo sendo que os fungos F13 (Fusarium) e F14 (Phoma) degradaram o óleo bruto em 24 horas. As dez linhagens testadas apresentaram oxidação biológica do meio 
em até 76 horas. Neste estudo foi sugerida a formação de um consórcio microbiano com os fungos que apresentaram melhores tempos de degradação em até 48 horas, sendo eles: F01, F06, F13, F14, F19, F23 e F27. Assim, o teste de biodegrabilidade do DCPIP se mostrou eficaz na seleção de espécies promissoras para a produção de consórcios fúngicos que degradam compostos orgânicos de petróleo e derivados possibilitando emprego em tratamentos por biorremediação de ambientes aquáticos.

\section{REFERÊNCIAS BIBLIOGRÁFICAS}

ARAÚJO, F. S.; LEMOS, J. L. Isolamento e identificação de fungos degradadores de petróleo. In: X Jornada de Iniciação Científica, Centro de Tecnologia MineralCETEM/MCT, 2002.

AZEVEDO, J.L. e COSTA, S. O. P. Exercícios práticos de Genética. São Paulo: Editora Nacional, 288p, 1973.

BISOGNIN, R. P. Análise do potencial microbiano de uma biopilha na biorremediação de solos contaminados por hidrocarbonetos. Dissertação (Mestrado em Tecnologia Ambiental) - Universidade de Santa Cruz do Sul - UNISC: Santa Cruz do Sul, 139p., 2012.

CRAPEZ, M. C.; BORGES, A. L. N.; BISPO, M. G. S.; PEREIRA, O. C. Tratamento para derrames de petróleo: biorremediação. Ciênc. Hoje, v. 30, n.79, p. 33-37, 2002.

GHAZALI, F. M.; RAHMAN, R. N. Z. A.; BASRI, M. Biodegradation of hydrocarbons in soil by microbial consortium. Intern. Biodet. \& Biodeg., v.54,p.61-67, 2004.

HANSON, K.G.; DESAI, J. D.; DESAI, A. J. A rapid and sample screening technique for potential crude oil degrading microorganisms. Biotec. Tech. v. 07, p. 745-748, 1993.

JACQUES, R. J. S.; BENTO, F. M.; ANTONIOLLI, Z. I.; CAMARGO; F. A. O. Biorremediação de solos contaminados com hidrocarbonetos aromáticos policíclicos. Ciênc. Rur., v.37, nº4, p. 1192-1201, 2007.

LEAHY, J. C.; COLWELL, R. R. Microbial degradation of hydrocarbons in the environment. Microb. Rev., v.54, n.3, p.305-315, 1990.

MARIANO, A. P. Avaliação do potencial de biorremediação de solos e águas subterrâneas contaminados com óleo diesel. Tese (Doutorado em Geociências e Meio Ambiente) Instituto de Geociências e Ciências Exatas, Universidade Estadual Paulista - Unesp: Rio Claro, SP, 162p., 2006.

PEIXOTO, R. S.; ROSADO, A. S.; TAKETANI, R. G. Bioprospecção da diversidade microbiana cultivável e não cultivável. In MELO, Itamar S. \& AZEVEDO, João L. Microbiologia Ambiental. $2^{\text {a }}$ Ed. Embrapa Meio Ambiente, 2008.

PEREIRA, J. A. Fungos endofíticos dos hospedeiros tropicais Stylosanthes guianensis e Musa cavendish. Tese (Doutorado em Fitopatologia) - Escola Superior de Agricultura Luiz de Queiroz: Piracicaba, 135p., 1993.

TONINI, R. M. C. W.; REZENDE, C. E. de; GRATIVOL, A. D. Degradação e Biorremediação de Compostos do Petróleo por Bactérias: Revisão. Oecol. Aust., 14(4): 1027-1035, 2010.

VAN HAMME, J. D.; ODUMERU, J. A.; WARD, O. P. Community dynamics of a mixedbacterial culture growing on petroleum hydrocarbons in batch culture. Can. $J$. Microbiol., v. 46, p.441-50, 2000. 